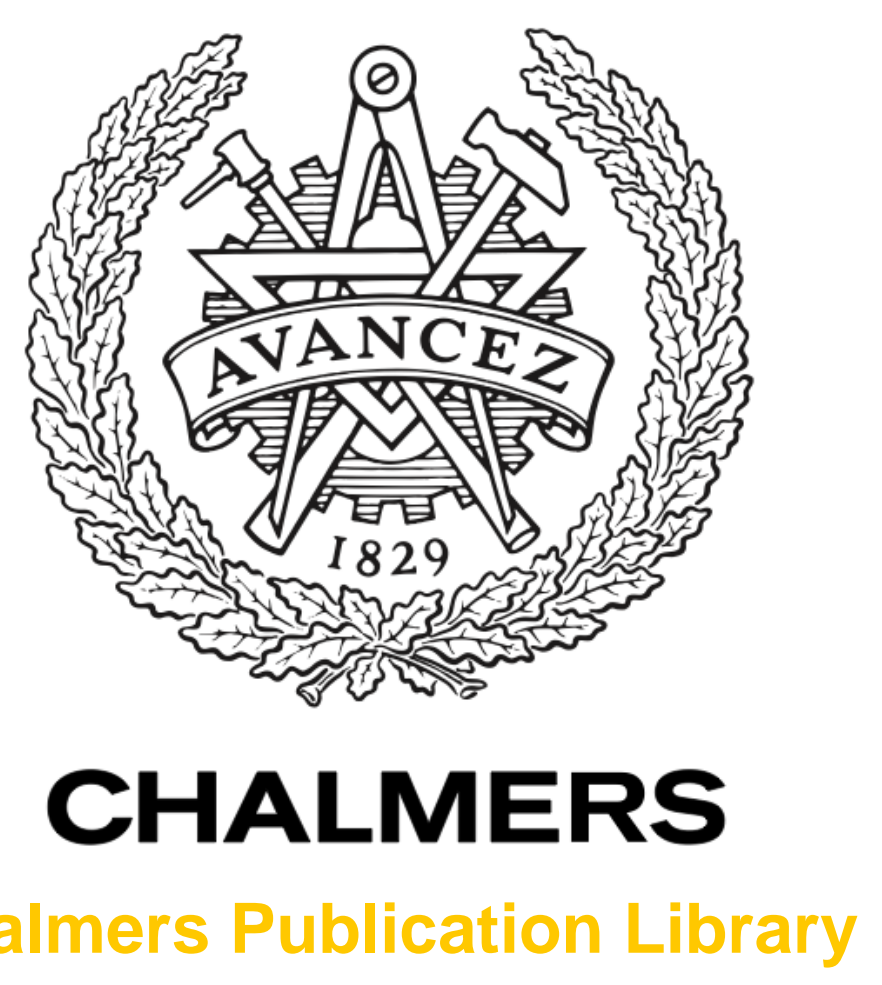

Chalmers Publication Library

Frequency determination in control applications: excitation-based approach

This document has been downloaded from Chalmers Publication Library (CPL). It is the author's version of a work that was accepted for publication in:

Proceedings of the Institution of mechanical engineers. Part l, journal of systems and control engineering (ISSN: 0959-6518)

Citation for the published paper:

Stotsky, A. (2012) "Frequency determination in control applications: excitation-based approach". Proceedings of the Institution of mechanical engineers. Part I, journal of systems and control engineering, vol. 226(8), pp. 1142-1148.

http://dx.doi.org/10.1177/0959651812447146

Downloaded from: http://publications.lib.chalmers.se/publication/165124

Notice: Changes introduced as a result of publishing processes such as copy-editing and formatting may not be reflected in this document. For a definitive version of this work, please refer to the published source. Please note that access to the published version might require a subscription. 


\title{
Frequency Determination in Control Applications: Excitation Based Approach *
}

\author{
Alexander Stotsky* \\ * Signals \& Systems, Chalmers University of Technology, Gothenburg, \\ SE-412 96, Sweden (e-mail: alexander.stotsky@chalmers.se)
}

\begin{abstract}
New algorithms for estimation of the frequencies of oscillating waveform signals are described. Model of the signals is presented in the form of linear difference equation with unknown coefficients, which define the frequencies and amplitudes. Coefficients are estimated utilizing the property of the persistence of excitation of oscillating signals. Exponentially damped and oscillating signals are described in a unified framework. A property of excitation is proved for exponentially damped signal that contains a single frequency via diagonal dominance of an information matrix. Two applications of this frequency estimation technique are considered. The first one is filtering of the wind speed signal in wind turbine control applications, and the second one is the frequency estimation of exponentially damped signal motivated by the engine knock detection applications.
\end{abstract}

Keywords: Frequency estimation, Persistence of excitation, Strictly diagonally dominant matrix, Exponentially damped signal, Projection algorithm, Wind speed model, Engine knock frequency

\section{INTRODUCTION}

Oscillating waveform signals appear in a large number of applications, including active noise, vibration and rotating machinery control, radar/sonar, seismic, audio and biomedical signal processing and many others. Estimation of unknown frequencies (frequency contents) of oscillating signals is an important problem of digital signal processing which was addressed in many papers.

A discrete-time adaptive estimation algorithm for a multifrequency signal based on the gradient update law and notch filter was proposed in [1]. A convergence analysis was performed using associated differential equation [2], [3] which proves a local stability of the system. This paper was the basis for a globally convergent continuous time frequency estimator of a single frequency based on classical adaptive control methods [4]. Continuous time version of algorithm proposed in [1] is also described in [5]. Recent algorithms of identification of a single frequency are based on the internal model principle [6], where the model is transformed and linearized with respect to unknown frequency [7],[8],[9],[10]. The method is well-discussed for both continuous and discrete time cases as well as for exponentially damped signals [11], [12].

Many, if not all, oscillating signals are sufficiently rich (persistently exciting). Therefore one of the most promising approaches to frequency estimation in real time applications is adaptive estimation techniques, where the parameter convergence is guaranteed by the persistence of excitation. This often simplifies an estimator and makes it very attractive for real-time applications. An excitation based adaptive technique for estimation of a continuoustime multi-frequency signal was proposed in [13] and ex-

* This work was supported by the Swedish Wind Power Technology Center tended further in [14], [15].

Discrete time estimation of multi-frequency signals is considered in this paper in a unified framework for both oscillating and exponentially damped signals. The properties of an information matrix (such as strict diagonal dominance) are in the main focus of this paper. These properties are important for both algorithm design and stability analysis and might be used in different ways depending on a model of the signal, which in turn, is application oriented.

Two models of the oscillating signals are considered in this paper. The first one is specified in the form of trigonometric polynomial (a sum of sines and cosines of known frequencies) with unknown coefficients. This model is wellsuited for frequency domain system identification, where the frequency response of dynamical system is analyzed via estimation of the parameters [16],[17], and the convergence of these parameters is guaranteed by the persistence of excitation [18]. The regressor vector consists of trigonometric functions (sines and cosines) at different frequencies in this case and corresponding information matrix is an SDD matrix $^{1}$ which allows application of new fast convergent algorithms for parameter estimation [20].

The second model that describes oscillating signal is a linear difference equation with unknown coefficients to be estimated. All the roots of the corresponding characteristic polynomial of this equation are complex and divided into conjugate pairs, where each pair defines the frequency and amplitude at this frequency. A regressor vector consists of the past values of the output in this case, and also a persistently exciting due to the oscillating properties of the signal [13]. Those two models described above are

\footnotetext{
1 A matrix is said to be a strictly diagonally dominant (SDD) matrix if in every row of the matrix, the magnitude of the diagonal entry in that row is larger than the sum of the magnitudes of all the other (non-diagonal) entries in that row [19]
} 
related via the solution of difference equation which contains trigonometric functions. However, the properties of information matrices based on different regressor vectors are also different for those two models. It is proved in this paper that the information matrix of the second model is an SDD matrix for single-frequency signals and the properties of this matrix should be further studied for the case of multi-frequency signals.

Two new applications of the frequency estimation technique based on the persistence of excitation are described in this paper in a unified framework. The first one is related to reduction of the wind speed model in wind turbine control applications, and the second one is the frequency estimation of exponentially damped signal (EDS) motivated by the engine knock detection applications.

The paper is organized as follows. Excitation based parameter estimation technique is described in the next Section followed by a new filtering technique of the wind speed signal in the wind turbine application, and frequency estimation of EDS in Sections 3 and 4 respectively. The paper ends with some discussions in Section 5.

\section{FREQUENCY ESTIMATION: EXCITATION BASED APPROACH}

Consider the following oscillating signal described by linear difference equation with known order and unknown coefficients:

$$
y_{k}=-a_{n-1} y_{k-1}-\ldots-a_{1} y_{k-n+1}-a_{0} y_{k-n}+c_{0}
$$

where $k=n, n+1, \ldots$. This equation has the following characteristic polynomial:

$$
\prod_{i=1}^{n}\left(z-z_{i}\right)=z^{n}+a_{n-1} z^{n-1}+\ldots+a_{1} z+a_{0}
$$

where $z_{i}$ are the roots of polynomial (2), and $a_{0}=$ $(-1)^{n} z_{1} \ldots z_{n}, \ldots, a_{n-1}=-\left(z_{1}+\ldots+z_{n}\right)$, are unknown coefficients of equation (1), and coefficient $c_{0}$ determines a bias of the signal.

If all the roots are complex and divided into $m=\frac{n}{2}$ conjugate pairs then each pair defines the frequency and amplitude, and equation (1) describes multi-frequency oscillating signal. Indeed, the solution of equation (1) can be written in this case as follows:

$$
y_{k}=b_{0}+\sum_{i=1}^{m} \rho_{i}^{k}\left[c_{i 1} \cos \left(q_{i} k\right)+c_{i 2} \sin \left(q_{i} k\right)\right]
$$

where $q_{i}$ is the frequency, $m$ is the number of frequencies, $\rho$ is the damping factor, and the coefficients $c_{i 1}, c_{i 2}$ and $b_{0}$ are determined from the initial conditions and the coefficients of equation (1) respectively.

Equation (3) is clearly suitable for description of oscillating signals with $\rho_{i}=1$ and the roots of (2) on the unit circle, and exponentially damped signals with $\rho_{i}<1$ and the roots inside of the unit circle.

Assuming that model (3) with $\rho_{i}=1$ has known frequencies and unknown coefficients, it can be used for frequency domain system identification and can be written in the following vector form:

$$
y_{k}=\psi_{k}^{T} \vartheta_{*}
$$

where $\psi_{k}$ is the regressor vector

$$
\begin{gathered}
\psi_{k}^{T}=\left[1 \cos \left(q_{1} k\right) \sin \left(q_{1} k\right) \cos \left(q_{2} k\right)\right. \\
\left.\sin \left(q_{2} k\right) \ldots \cos \left(q_{m} k\right) \sin \left(q_{m} k\right)\right]
\end{gathered}
$$

and $\vartheta_{*}$ is a corresponding vector of constant unknown parameters. An information matrix $B=\sum_{j=k}^{j=k+w} \psi_{j} \psi_{j}^{T}$ with regressor (5) is an SDD matrix for a sufficiently large window size $w$. This matrix has the following property $\left\|I-D^{-1} B\right\|_{\infty} \leq \kappa<1$, (where $D$ is a diagonal matrix with diagonal elements of matrix $B, \kappa>0$, and the norm $\|\cdot\|_{\infty}$ is the maximum row sum matrix norm, and $I$ is the identity matrix) which allows the design of fast, robust and computationally efficient algorithms for estimation of unknown parameters [20]. Model (4) is suitable for a frequency domain system identification for example, but the frequencies of the signal can not be estimated using this model since regressor $\psi_{k}$ contains known trigonometric functions.

Frequencies of the signal can be estimated using equation (1) which can be written in the following form:

$$
y_{k}=\varphi_{k}^{T} \theta_{*}
$$

where $\varphi_{k}$ is the regressor and $\theta_{*}$ is the vector of constant unknown parameters defined as follows:

$$
\begin{aligned}
& \varphi_{k}^{T}=\left[\begin{array}{lllll}
y_{k-1} & \ldots & y_{k-n+1} & y_{k-n} & 1
\end{array}\right] \\
& \theta_{*}^{T}=\left[\begin{array}{lllll}
-a_{n-1} & \ldots & -a_{1} & -a_{0} & c_{0}
\end{array}\right]
\end{aligned}
$$

The model of signal (6) can be introduced as follows:

$$
\hat{y}_{k}=\varphi_{k}^{T} \theta_{k}
$$

where $\theta_{k}$ is the vector of adjustable parameters.

It was first discovered in [13] that regressor $\varphi_{k}$, defined in (7) is persistently exciting i.e., there exist positive constants $r_{0}, r_{1}$ and $w$ such that the following inequality holds:

$$
0<r_{0} I \leq \sum_{j=k}^{j=k+w} \varphi_{j} \varphi_{j}^{T} \leq r_{1} I
$$

where $I$ is the identity matrix. However, an information matrix $\sum_{j=k}^{j=k+w} \varphi_{j} \varphi_{j}^{T}$ in (10) is an SDD matrix for a singlefrequency signal only. This matrix is not an SDD matrix for multi-frequency signals and therefore fast and computationally efficient algorithms mentioned above can not be applied for parameter estimation in this case. Properties of this matrix should be further studied that might lead to the performance improvement of the excitation based estimation algorithms.

Many algorithms can be applied for estimation of the parameter vector $\theta_{*}$, where the parameter convergence of $\theta_{k}$ to $\theta_{*}$ is guaranteed by excitation (10). One of such algorithms is a simple Kaczmarz algorithm where the output of the model matches the measured signal exactly in each discrete step and the vector of the parameter mismatch is orthogonal to the regressor vector. A computational simplicity and robustness against measurement noise make Kaczmarz algorithm very attractive for estimation of the frequency contents of complex signals with a large number 
of frequencies in real-time applications. A parameter convergence is guaranteed by the property of the persistence of excitation (10). The roots of polynomial (2) can be calculated as soon as its coefficients are identified and the frequencies of the signal can then be calculated.

Kaczmarz algorithms are summarized in Table 1 where a classical Kaczmarz algorithm modified with the gain matrix $\Gamma_{k}$ is shown on the top of the Table. Three items are listed for the gain update algorithms. The first item where the gain matrix is equal to identity matrix corresponds to a classical Kaczmarz algorithm [21], and the second and third ones describe Kaczmarz algorithms modified with least-squares gain updates [20],[22],[23]. The third item shows the least-squares gain update with a forgetting factor and forgetting factor of the second one is equal to one.

These algorithms are applied to the frequency determination in the next Sections.

\begin{tabular}{|c|c|}
\hline \multicolumn{1}{|c|}{ KACZMARZ ALGORITHMS } \\
$\theta_{k}=\theta_{k-1}+\frac{\Gamma_{k-1} \varphi_{k}}{\varphi_{k}^{T} \Gamma_{k-1} \varphi_{k}}\left(y_{k}-\theta_{k-1}^{T} \varphi_{k}\right)$ \\
\hline $\mathrm{N}$ & GAIN UPDATE ALGORITHM $\Gamma_{k}$ \\
\hline 1 & $\Gamma_{k}=I \quad \begin{array}{l}\Gamma_{k}^{-1}=\Gamma_{k-1}^{-1}+\varphi_{k} \varphi_{k}^{T} \\
2\end{array}$ \\
\hline $\begin{array}{l}\Gamma_{k}=\Gamma_{k-1}-\frac{\Gamma_{k-1} \varphi_{k} \varphi_{k}^{T} \Gamma_{k-1}}{1+\varphi_{k}^{T} \Gamma_{k-1} \varphi_{k}}, \\
\Gamma_{0}=\frac{1}{\gamma_{0}} I, \quad \gamma_{0}>0\end{array}$ \\
\hline 3 & $\begin{array}{l}\Gamma_{k}^{-1}=\lambda_{0} \Gamma_{k-1}^{-1}+\lambda_{1} \varphi_{k} \varphi_{k}^{T}, \\
\Gamma_{k}=\frac{1}{\lambda_{0}}\left[\Gamma_{k-1}-\frac{\Gamma_{k-1} \varphi_{k} \varphi_{k}^{T} \Gamma_{k-1}}{\left(\frac{\lambda_{0}}{\lambda_{1}}+\varphi_{k}^{T} \Gamma_{k-1} \varphi_{k}\right)}\right] \\
\Gamma_{0}=\frac{1}{\gamma_{0}} I, \quad \gamma_{0}>0, \quad 0<\lambda_{0} \leq 1, \quad \lambda_{1}>0\end{array}$ \\
\hline
\end{tabular}

Table 1. A family of Kaczmarz algorithms

\section{WIND SPEED MODEL REDUCTION: EXCITATION BASED FILTERING}

Future laser sensor technologies will be capable of measuring wind speed at a distance in front of the wind turbine with relatively high sample rates [24]. Wind speed signal measured at a distance in front of the turbine allows preprocessing and generation of a high quality feedforward control signal [25]. A time series model in the form of (1) is widely used for prediction of wind speed oscillations at a distance in front of the wind turbine (see for example [26] and [27] and references therein). A low frequency component should be extracted from the noise contaminated wind speed measurements in front of the turbine to achieve a high performance turbine regulation. A low pass filtering which the simplest solution in this case introduces a phase lag and might have negative impact on the power efficiency of the turbine. Excitation based filtering of the wind speed

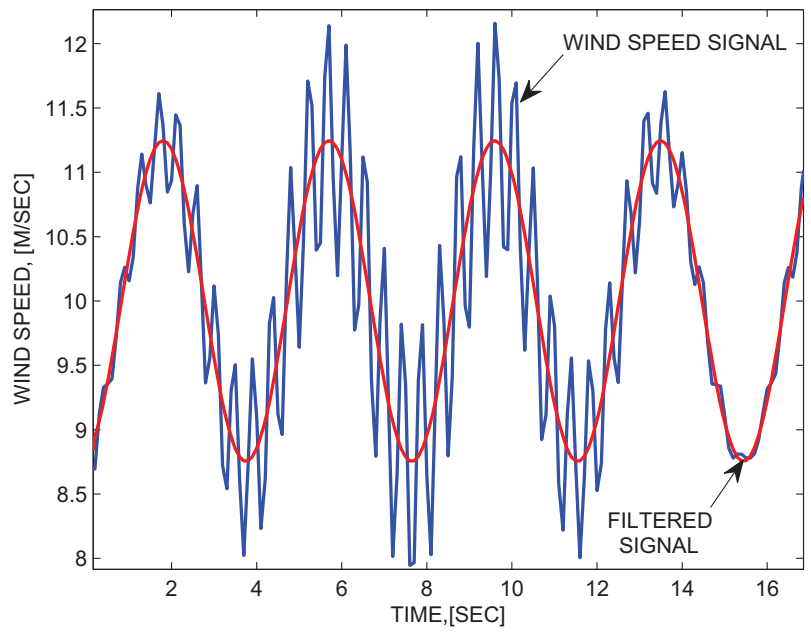

FIG 1. Filtering of the wind speed signal. The wind speed signal that contains three frequencies is plotted with a blue line. Frequencies are identified using the approach described in Section 3. The lowest frequency is selected for the filtered signal plotted with a red line.

signal, described in this paper provides a computationally efficient solution without a phase lag. It is assumed that the wind speed is described by linear difference equation (1) with unknown coefficients. Wind speed model is specified in the form of (9) with the parameters adapted via Kaczmarz algorithm $\theta_{k}=\theta_{k-1}+\frac{\Gamma_{k-1} \varphi_{k}}{\varphi_{k}^{T} \Gamma_{k-1} \varphi_{k}}\left(y_{k}-\theta_{k-1}^{T} \varphi_{k}\right)$, where a gain matrix $\Gamma_{k}$ is adjusted via the second algorithm in Table 1 . As soon as the coefficients of polynomial (2) are found, its complex roots are calculated. Those roots define the frequencies and amplitudes at each frequency. Filtering of the wind speed signal is performed via a selection of the lowest frequency. As soon as the low frequency component of the wind speed signal is extracted, its derivative which is taken analytically can be used in the feedforward part of the turbine speed controller [25].

This filtering approach is illustrated in Figure 1, which shows the wind speed signal that contains three frequencies filtered via excitation based filtering.

\section{FREQUENCY DETERMINATION OF EXPONENTIALLY DAMPED SIGNAL}

Determination of the frequencies of EDS is motivated by the engine signal processing applications [28]. A knock detection circuit that is based on the signal of an accelerometer installed on the engine block of a spark ignition automotive engine has a band-pass filter with a certain frequency as a parameter to be calibrated. A determination of the frequency which is the most suitable for the knock detection is very important for real-time applications. In the case of knocking an accelerometer reads exponentially vanishing oscillating signal. Similar signals also appear in seismic and audio signal processing applications. Notice that exponentially damped signals are exciting over some finite intervals only, and sufficient intensity is required for frequency estimation. 


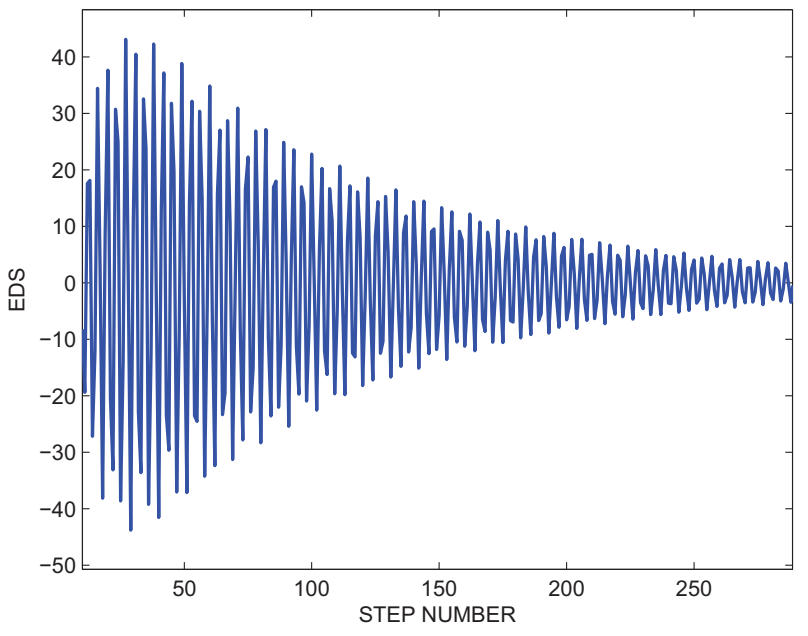

FIG 2. Exponentially damped signal that contains three frequencies.

Consider the following model of damped signal that contains a single frequency $q$ :

$$
y_{k}=c_{1} \rho^{k-1} \cos (q(k-1))+c_{2} \rho^{k-1} \sin (q(k-1))
$$

where $c_{1}$ and $c_{2}$ are constants determined from the initial conditions, $k=1,2 \ldots$ and $\rho<1$. Model (11) can be written in form (6) with

$$
\varphi_{k}^{T}=\left[\begin{array}{ll}
y_{k-1} & y_{k-2}
\end{array}\right], \quad \theta_{*}^{T}=\left[\begin{array}{ll}
-a_{1} & -a_{0}
\end{array}\right]
$$

Information matrix $A=\sum_{k=3}^{w} \varphi_{k} \varphi_{k}^{T}$ is defined as follows:

$$
A=\left[\begin{array}{cc}
\underbrace{\sum_{k=3}^{w} y_{k-1}^{2}}_{a_{11}} & \underbrace{\sum_{k=3}^{w} y_{k-1} y_{k-2}}_{a_{12}} \\
\underbrace{\sum_{k=3}^{w} y_{k-1} y_{k-2}}_{a_{21}} & \underbrace{\sum_{k=3}^{w} y_{k-2}^{2}}_{a_{22}}
\end{array}\right]
$$

Elements of this matrix are calculated explicitly and listed in Appendix. All the elements have average parts and periodic parts. An average part of the matrix $A$ for $\rho$ which is sufficiently close to one and for a sufficiently large $w$ (so that the following is true $\rho^{2(w-2)+2} \approx \rho^{2(w-3)+2}$ ) can be written as follows:

$$
A=\frac{1}{2}\left(c_{1}^{2}+c_{2}^{2}\right) \frac{1-\rho^{2(w-3)+2}}{1-\rho^{2}}\left[\begin{array}{cc}
1 & \rho \cos (q) \\
\rho \cos (q) & 1
\end{array}\right]
$$

which shows that matrix $A$ an SDD matrix.

Adjustable model of signal (6) is specified in form (9) with the parameters updated via Kaczmarz algorithm $\theta_{k}=\theta_{k-1}+\frac{\Gamma_{k-1} \varphi_{k}}{\varphi_{k}^{T} \Gamma_{k-1} \varphi_{k}}\left(y_{k}-\theta_{k-1}^{T} \varphi_{k}\right)$, where a gain matrix $\Gamma_{k}$ is adjusted via the second algorithm in Table 1 as follows:

$$
\Gamma_{k}^{-1}=\Gamma_{k-1}^{-1}+\varphi_{k} \varphi_{k}^{T}, \quad \Gamma_{0}^{-1}=\gamma_{0} I, \quad \gamma_{0}>0
$$

This sequence of matrices $\Gamma_{k}^{-1}, k=1,2,3, \ldots, w$ can be written as follows:

$$
\begin{aligned}
& \Gamma_{0}^{-1}=\gamma_{0} I \\
& \Gamma_{1}^{-1}=\Gamma_{0}^{-1}+\varphi_{1} \varphi_{1}^{T} \\
& \Gamma_{2}^{-1}=\Gamma_{1}^{-1}+\varphi_{2} \varphi_{2}^{T} \\
& \cdots \quad \quad \cdots \\
& \Gamma_{w}^{-1}=\Gamma_{w-1}^{-1}+\varphi_{w} \varphi_{w}^{T}
\end{aligned}
$$

Direct substitution yields:

$$
\begin{gathered}
\underbrace{\Gamma_{w}^{-1}}_{\text {inverse of gain matrix }}=\underbrace{\gamma_{0} I}_{\text {initial matrix }} \\
+\underbrace{\sum_{k=1}^{\sum_{k=1} \varphi_{k} \varphi_{k}^{T}}}_{\text {information matrix } A}
\end{gathered}
$$

Relation (14) shows that the matrix $\Gamma_{k}^{-1}$ can be made an SDD matrix for several initial steps $k=0,1,2, \ldots, w$ with a sufficiently large and positive $\gamma_{0}$ since all the elements of $\sum_{k=1}^{w} \varphi_{k} \varphi_{k}^{T}$ are bounded. For a sufficiently large $w$ the matrix $\sum_{k=1}^{w} \varphi_{k} \varphi_{k}^{T}$ becomes an SDD matrix with positive diagonal entries. Therefore the matrix $\Gamma_{k}^{-1}$ is also an SDD matrix for $k=w, \ldots \infty$ since an addition of the initial matrix $\gamma_{0} I, \gamma_{0}>0$ does not destroy a diagonal dominance. In other words a sufficiently large $\gamma_{0}$ ensures a diagonal dominance until the information matrix $\sum_{k=1}^{w} \varphi_{k} \varphi_{k}^{T}$ becomes diagonally dominant. Therefore the matrix $\Gamma_{k}^{-1}$ is a positive definite matrix in all the steps $k=0,1,2, \ldots$ since it is an SDD symmetric matrix with all positive diagonal entries.

Therefore, due to arguments similar to [18] the following transient bound can be established for the estimation error $\tilde{\theta}_{k}=\theta_{k}-\theta_{*}$ :

$$
\left\|\tilde{\theta}_{k}\right\| \leq \sqrt{\frac{V_{0}}{\lambda_{\min }\left(\Gamma_{k}^{-1}\right)}}
$$

where $V_{0}=\left(\theta_{0}-\theta_{*}\right)^{T} \Gamma_{0}^{-1}\left(\theta_{0}-\theta_{*}\right)$ and $\lambda_{\min }\left(\Gamma_{k}^{-1}\right)$ is a minimal eigenvalue of the matrix $\Gamma_{k}^{-1}$. This upper bound can be made sufficiently small since a minimal eigenvalue of the matrix $\Gamma_{k}^{-1}$ increases with a step number and rapidly reaches a large steady-state value.

Frequency estimation of a single-frequency exponentially damped signal is plotted in Figure 3, and the frequency estimation of the signal that contains three frequencies plotted in Figure 2, using estimation algorithm described above is shown in Figure 4.

\section{DISCUSSION}

Richness of oscillating signals can successfully be used for frequency estimation. Those signals are modeled using linear difference equations with unknown coefficients, which define the frequencies and amplitudes. The convergence of estimated coefficients to their true values is guaranteed by the persistence of excitation that also simplifies estimation 


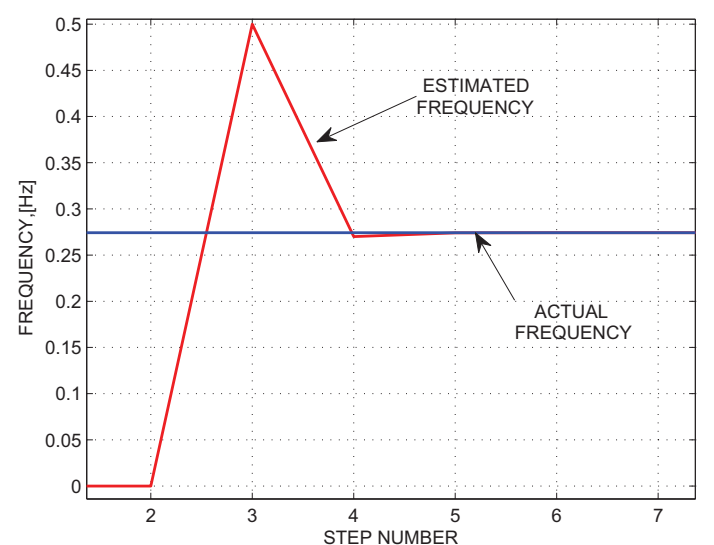

FIG 3. Frequency estimation of EDS that contans a single frequency with the second algorithm in Table 1 described in Section 2. Estimated frequency is plotted with a red line and actual frequency is plotted with a blue line.

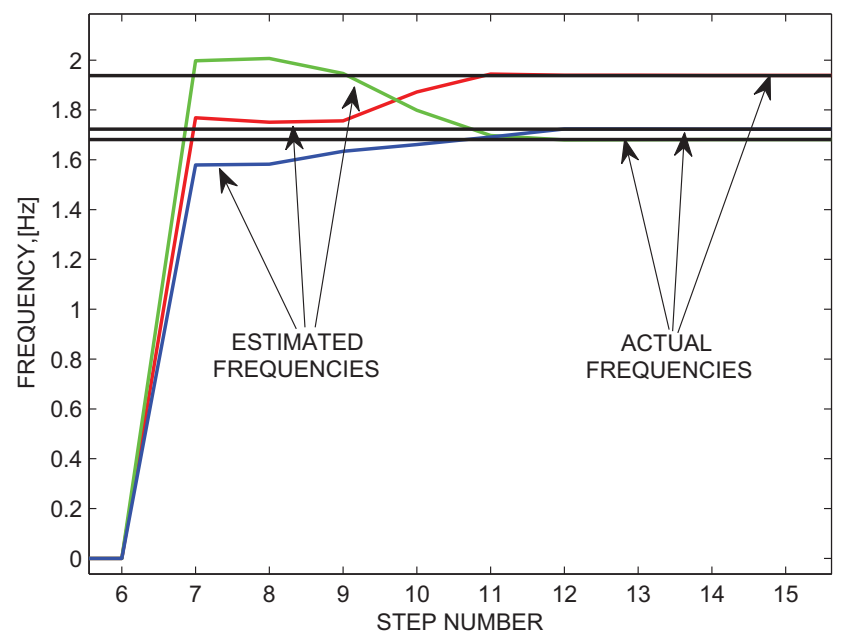

FIG 4. Frequency estimation of the signal plotted in Figure 2 with the third algorithm in Table 1, described in Section 2. Three estimated frequencies are plotted with blue, red and green lines. Actual frequencies are plotted with black lines.

algorithms. Strictly diagonally dominance of the information matrix together with the property of persistence of excitation might successfully be used for the performance improvement of existing estimation algorithms. It is shown that information matrix (in the case of frequency estimation) is an SDD matrix for single-frequency signals only. Information matrix is not an SDD matrix in the case where the number of frequencies is greater than or equal to two. The properties of this information matrix and gain matrix $\Gamma_{k}$ with different forgetting factors (see Table 1) should be further studied for multi-frequency signals, aiming to the performance improvement of the excitation based estimation algorithms.

Besides, frequency determination using this technique requires very high accuracy of estimation of the coefficients of the difference equations. Such an accuracy can not be achieved in the presence of unmodeled frequencies and significant measurement noise. Therefore this estimation technique should be applied together with the preprocessing methods which can clean up the signal sufficiently. On the contrary the coefficients of the trigonometric polynomials with known frequencies in frequency domain identification can be estimated with acceptable accuracy in the presence of significant measurement noise and unmodeled frequencies.

\section{APPENDIX}

Elements of the matrix $A$ are evaluated explicitly using the following relations:

$$
\begin{aligned}
& \sum_{k=n}^{w} \rho^{2(k-n)}=\frac{1-\rho^{2(w-n)+2}}{1-\rho^{2}} \\
& \sum_{k=n}^{w} \rho^{2(k-n)} \cos (2(k-n) q)=\frac{1-\rho^{2} \cos (2 q)}{1+\rho^{4}-2 \rho^{2} \cos (2 q)} \\
+ & \frac{-\rho^{2(w-n)+2} \cos ((2(w-n)+2) q)}{1+\rho^{4}-2 \rho^{2} \cos (2 q)} \\
+ & \frac{\rho^{2(w-n)+4} \cos (2 q(w-n))}{1+\rho^{4}-2 \rho^{2} \cos (2 q)} \\
& \sum_{k=n}^{w} \rho^{2(k-n)} \sin (2(k-n) q)=\frac{\rho^{2} \sin (2 q)}{1+\rho^{4}-2 \rho^{2} \cos (2 q)} \\
+ & \frac{-\rho^{2(w-n)+2} \sin ((2(w-n)+2) q)}{1+\rho^{4}-2 \rho^{2} \cos (2 q)} \\
+ & \frac{\rho^{2(w-n)+4} \sin (2 q(w-n))}{1+\rho^{4}-2 \rho^{2} \cos (2 q)}
\end{aligned}
$$

for $w \geq n, n=1,2, \ldots$, where $w$ is a window size and $n$ is a parameter, and

$$
\begin{aligned}
& a_{11}=\sum_{k=3}^{w} y_{k-1}^{2}=\underbrace{\frac{1}{2}\left(c_{1}^{2}+c_{2}^{2}\right) \frac{1-\rho^{2(w-2)+2}}{1-\rho^{2}}}_{\text {average part }} \\
& +\frac{1}{2}\left(c_{1}^{2}-c_{2}^{2}\right)\left[\frac{1-\rho^{2} \cos (2 q)}{1+\rho^{4}-2 \rho^{2} \cos (2 q)}\right. \\
& +\frac{-\rho^{2(w-2)+2} \cos ((2(w-2)+2) q)}{1+\rho^{4}-2 \rho^{2} \cos (2 q)} \\
& \left.+\frac{\rho^{2(w-2)+4} \cos (2 q(w-2))}{1+\rho^{4}-2 \rho^{2} \cos (2 q)}\right] \\
& +c_{1} c_{2}\left[\frac{\rho^{2} \sin (2 q)-\rho^{2(w-2)+2} \sin ((2(w-2)}{1+\rho^{4}-2 \rho^{2} \cos (2 q)}+2\right) \\
& \left.+\frac{\rho^{2(w-2)+4} \sin (2 q(w-2))}{1+\rho^{4}-2 \rho^{2} \cos (2 q)}\right]-c_{1}^{2} \\
& +\frac{-\rho^{2(w-3)+2} \cos ((2(w-3)+2) q)}{1+\rho^{4}-2 \rho^{2} \cos (2 q)} \\
& +\frac{1}{2}\left(c_{1}^{2}-c_{2}^{2}\right)\left[\frac{1-\rho^{2} \cos (2 q)}{1+\rho^{4}-2 \rho^{2} \cos (2 q)}\right. \\
& a_{22}=\sum_{k=3} y_{k-2}^{2}=\frac{1}{2\left(c_{1}^{2}+c_{2}^{2}\right) \frac{1-\rho^{2(w-3)+2}}{1-\rho^{2}}} \\
& +\frac{1 v e r a g e \text { part }}{2(w)}
\end{aligned}
$$




$$
\begin{aligned}
+ & \left.\frac{\rho^{2(w-3)+4} \cos (2 q(w-3))}{1+\rho^{4}-2 \rho^{2} \cos (2 q)}\right] \\
+ & c_{1} c_{2}\left[\frac{\rho^{2} \sin (2 q)-\rho^{2(w-3)+2} \sin ((2(w-3)+2) q)}{1+\rho^{4}-2 \rho^{2} \cos (2 q)}\right. \\
+ & \left.\frac{\rho^{2(w-3)+4} \sin (2 q(w-3))}{1+\rho^{4}-2 \rho^{2} \cos (2 q)}\right] \\
& =\frac{1}{2}\left(c_{1}^{2}+c_{2}^{2}\right) \rho \cos (q) \frac{1-\rho^{2(w-3)+2}}{1-\rho^{2}} \\
& a_{12}=a_{21}=\sum_{k=3} y_{k-1} y_{k-2} \\
+ & \frac{1}{2}\left(c_{1}^{2}-c_{2}^{2}\right)\left[\frac{\rho \cos (q)-\rho^{2 w-3} \cos ((2 w-3) q)}{1+\rho^{4}-2 \rho^{2} \cos (2 q)}\right. \\
+ & \left.\frac{-\rho^{3} \cos (q)+\rho^{2 w-1} \cos ((2 w-5) q)}{1+\rho^{4}-2 \rho^{2} \cos (2 q)}\right] \\
+ & c_{1} c_{2}\left[\frac{\rho \sin (q)-\rho^{2 w-3} \sin ((2 w-3) q)}{1+\rho^{4}-2 \rho^{2} \cos (2 q)}\right. \\
+ & \left.\frac{\rho^{3} \sin (q)+\rho^{2 w-1} \sin ((2 w-5) q)}{1+\rho^{4}-2 \rho^{2} \cos (2 q)}\right]
\end{aligned}
$$

\section{REFERENCES}

[1] Regalia P., An Improved Lattice-Based IIR Notch Filter, IEEE Transactions on Signal Processing, vol. 39, pp. 2124-2128, Sept. 1991.

[2] Derevitsky D. and Fradkov A., Applied Theory of Discrete Time Systems, Moscow, Nauka, 1981, 216 pages, in Russian.

[3] Ljung L., Analysis of Recursive Stochastic Algorithms, IEEE Transactions on Automatic Control, vol. AC-22, pp. 551-575, Aug. 1977.

[4] Hsu L., Ortega R., and Damm G., A Globally Convergent Frequency Estimator, IEEE Transactions on Automatic Control, vol. 44, pp. 698-713, Apr. 1999.

[5] Bodson M. and Douglas S., Adaptive Algorithms for the Rejection of Periodic Disturbances with Unknown Frequencies, Automatica, vol. 33, No. 12, pp. 22132221, Dec. 1997.

[6] Nikiforov V., Adaptive Servomechanism Controller with an Implicit Reference Model, Int. J. Control, vol. 68, pp. 277-286, 1997.

[7] Aranovskii S., Bobtsov A., Kremlev A., and Lukyanova G., A Robust Algorithm for Identification of the Frequency of a Sinusoidal Signal, Journal of Computer and Systems Sciences International, 2007, vol. 46, No. 3, pp. 371-376, Pleiades Publishing, Ltd.

[8] Aranovskiy S., Bobtsov A., Kremlev A., Nikolaev N., and Slita O., Identification of Frequency of Biased Harmonic Signal, European Journal of Control, vol. 16, No. 2, pp. 129-139, Feb. 2010.

[9] Bobtsov A., New Approach to the Problem of Globally Convergent Frequency Estimator, International Journal of Adaptive Control and Signal Processing, vol.22, pp. 306-317, 2008.

[10] Zhang Q. and Brown L., Noise Analysis of an Algorithm for Uncertain Frequency Identification, IEEE
Transactions on Automatic Control, vol. 51, No. 1, Jan. 2006, pp. 103-110.

[11] Lu J. and Brown L., Identification of Exponentially Damped Sinusoidal Signals, Proceedings of the 17th World Congress The International Federation of Automatic Control Seoul, Korea, July 6-11, 2008, pp. 5089-5094.

[12] Lu J., Advances in Internal Model Principle Control Theory, PhD Thesis, The University of Western Ontario, London, Ontario, Canada, 2011, 145 pages.

[13] Marino R. and Tomei P., Global Estimation of n Unknown Frequencies, IEEE Transactions on Automatic Control, vol. 47, No. 8, August 2002, pp. 1324 - 1328.

[14] Xia X. Global Frequency Estimation Using Adaptive Identifiers, IEEE Transactions on Automatic Control, vol. 47, No. 7, July 2002, pp. 1188-1193.

[15] Hou M., Parameter Identification of Sinusoids, IEEE Transaction on Automatic Control, vol. 57, No. 2, Feb. 2012, pp. 467-472.

[16] Ljung L., System Identification: Theory for the User, Prentice Hall, 1999.

[17] Pintelon R. and Schoukens J., System Identification a Frequency Domain Approach, Piscataway, NJ: IEEE Press, ISBN 0-7803-6000-1, 2001.

[18] Stotsky A. A New Frequency Domain System Identification Method, Proceedings of the Institution of Mechanical Engineers, Part I: Journal of Systems and Control Engineering, vol. 226, 2012, pp. 111-124.

[19] Horn R. and Johnson C. Matrix Analysis, Cambridge University Press, 1985.

[20] Stotsky A. Recursive Trigonometric Interpolation Algorithms, Proceedings of the Institution of Mechanical Engineers, Part I: Journal of Systems and Control Engineering, vol. 224, No. 1, 2010, pp. 65-77.

[21] Astrom K. J., Wittenmark B., Adaptive Control, Addison-Wesley, 1989.

[22] Avedyan E., Modified Kaczmarz Algorithms for Estimating the Parameters of Linear Plants, Autom. Remote Control, vol. 39, No. 5, 1978, pp. 674-680.

[23] Goodwin G. and Sin K., Adaptive Filtering, Prediction and Control. Englewood Cliffs, NJ: Prentice-Hall, 1984.

[24] Wang N., Johnson K. and Wright A., LIDAR-based FX-RLS Feedforward Control for Wind Turbine Load Mitigation, American Control Conference on O'Farrell Street, San Francisco, CA, USA June 29 - July 01, 2011, pp. 1910-1915.

[25] Stotsky A. and Egardt B., Model Based Control of Wind Turbines: Look-Ahead Approach, Proc. of the 7-th IFAC Symposium on Robust Control Design, Aalborg, Denmark, June 20-22, 2012.

[26] Kusiak A., Song Z. and Zheng H., Anticipatory Control of Wind Turbines with Data-Driven Predictive Models, IEEE Transactions on Energy Conversion, vol. 24, No. 3, Sept. 2009, pp. 766-774.

[27] Sirdas S., Daily Wind Speed Harmonic Analysis for Marmara Region in Turkey, Energy Conversion and Management, vol.46, 2005, pp. 1267 - 1277.

[28] Stotsky A. Automotive Engines: Control, Estimation, Statistical Detection, Springer-Verlag, BerlinHeidelberg, 2009. 